\title{
Problem Solving and Motivation - Getting our Students in Flow
}

\author{
N. Sanjay Rebello \& Dean A. Zollman \\ Department of Physics, Kansas State University, 116 Cardwell Hall, Manhattan, KS 66506
}

\begin{abstract}
Csíkszentmihályi proposed the psychological concept of flow as signifying a state of complete involvement and enjoyment in an activity. When learners are in flow they are motivated, engaged, and completely focused on the task at hand, resulting in effortful learning. In this paper we explore the connections between the concept of flow and our model of transfer of learning as applied to problem solving. Our model of transfer purports two cognitive mechanisms horizontal and vertical - that learners use to construct knowledge. Further, it proposes that carefully designed sequences of horizontal and vertical learning which provide scaffolding within a learner's zone of proximal development can facilitate learners to navigate an optimal adaptability corridor and foster progress toward adaptive expertise as characterized by Bransford \& Schwartz. By exploring the connections between flow and our model of transfer, we hope to gain insights into what can motivate learners to become better problem solvers.
\end{abstract}

Keywords: transfer of learning, problem solving, adaptive expertise, flow

PACS: $01.40 . \mathrm{Fk}$

\section{INTRODUCTION}

Transfer of learning has traditionally been defined as applying what one has learned in one context to a different context. [1] Traditional models tend to focus on the cognitive aspects of transfer such as existence of identical elements [2] or similarities in deep structure. [3] Information processing models suggest that transfer requires abstract, symbolic mental representations. [4]

Contemporary models of transfer focus on more than just the cognitive aspects of transfer and have included several other mediating factors. These models account for aspects the traditional models neglect. [5-7] They take into account the socio-cultural factors that mediate transfer and view transfer from a learner's perspective rather than the researcher's perspective. Most importantly, they consider transfer as an active dynamic process of knowledge construction rather than merely knowledge activation.

In this paper we present a theoretical framework that consolidates traditional and contemporary perspectives on transfer of learning, in a way similar to that proposed by Schwartz and Bransford. [8] We then relate this model to the psychological concept of flow proposed by Csíkszentmihályi. [9]

\section{THEORETICAL FRAMEWORK}

As per our framework of transfer, which is based on a two-level model proposed by Redish, [10] transfer, at its most fundamental level, is a dynamic process involving the creation of associations. We propose qualitative two different kinds of transfer.
Horizontal transfer involves acquiring explicitly provided information and mapping it onto a pre-created knowledge structure that is aligned with this new information. If such alignment or assignment is not possible because the external problem representation does not match the learner's internal knowledge structure the learner reaches an impasse. Vertical transfer occurs when learners' prior knowledge structure does not align with new information. So, the learner constructs knowledge in situ through successive activation and suppression of associations between knowledge elements. The learner must choose between competing internal representations or construct a new one for this situation. Vertical transfer is a hallmark of most real-world problems.

Our ideas of horizontal and vertical transfer described above are not new. These two kinds of transfer mechanisms are analogous to those proposed by Bransford and Schwartz - Sequestered Problem Solving (SPS) and Preparation for Future Learning (PFL). [5] SPS focuses on whether students can directly apply their learning to a new situation without any scaffolding or support. PFL focuses on whether they are prepared to learn in the future by bringing to bear their earlier experiences to construct new knowledge.

More recently, Schwartz and Bransford suggested the notions of efficiency and innovation in transfer. [8] Efficiency, which is akin to horizontal transfer, refers to a learner's ability to rapidly recall and apply their knowledge in a new situation. Innovation, which is akin to vertical transfer, is their ability to restructure 
their thinking or reorganize the problem scenario so that it becomes more tractable than before.

Schwartz and Bransford also suggest that efficiency and innovation are both equally important goals. [8] For instance, we want students to be innovative and develop new strategies for solving new and previously unseen problems. However, we also want students to be efficient in solving previously seen problems so that they do not have to reinvent problem solving strategies each time they are faced with a problem. In other words, we want students to be adaptive learners who can distinguish between situations in which proven strategies can be applied and those in which they cannot be applied, necessitating the innovations of new strategies. Thus students must be guided along what they call an Optimal Adaptability Corridor (OAC) - a diagonal path as shown in Fig. 1 that simultaneously promotes both efficiency and innovation.

Above the OAC lies a region where the learner is being forced to become innovative before she is allowed to develop adequate efficiency. She is thus a "frustrated novice", who is confused about what she needs to do. Alternatively, below the OAC lies a region where the learner is more efficient than she is innovative. She is a "routine expert" who becomes bored with her tasks.

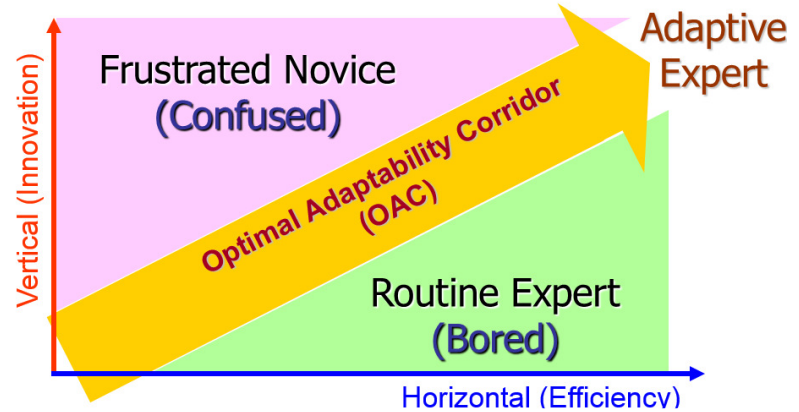

FIGURE 1. The OAC as proposed by Schwartz \& Bransford. [8]

\section{AFFECTIVE IMPLICATIONS OF FRAMEWORK AND ALIGNMENT WITH FLOW}

The notion of the OAC and the areas of confusion and boredom above and below the OAC allows us to recognize the affective implications of our transfer framework.

We find that these affective implications of our framework are further supported by recognizing how this model is similar the model of flow proposed by Csíkszentmihályi. [9]

Csíkszentmihályi proposed the psychological concept of flow as signifying a state of complete involvement and enjoyment in an activity. When learners are in flow they are motivated, engaged, and completely focused on the task at hand, resulting in effortful learning. The model is shown in Fig. 2.

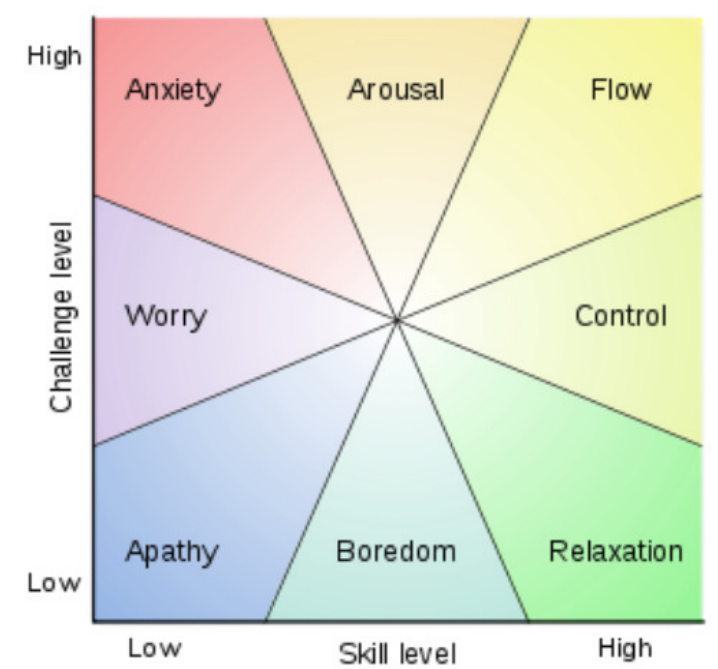

FIGURE 2. Model proposed by Csíkszentmihályi [9]

Note that the model shows eight affective domains on a two-dimensional continuum of skill and challenge. Skill is akin to horizontal transfer i.e. being efficient. Challenge is akin to vertical transfer i.e. being innovative.

The areas of confusion and boredom on Fig. 2 seem to overlap with the regions of anxiety and relaxation in Fig. 3 respectively. More importantly, the region of adaptive expertise in Fig. 2 seems to overlap with the area of flow in Fig. 3.

These connections between the two models seem to suggest that adaptive expertise has an affective component that is related to flow.

\section{SUMMARY \& IMPLICATIONS}

Overall, this paper demonstrates the alignment between our model of transfer and Csíkszentmihály’s model. The main implications of these connections are that as educators and researchers who study and promote transfer, we must attend to the affective aspects of this model.

\section{ACKNOWLEDGMENTS}

This work is supported in part by grants from U.S. National Science Foundation. Opinions expressed are those of the authors and not necessarily those of the foundation. 


\section{REFERENCES}

1. N.S. Rebello et. al, presented at Annual Meeting of the National Association for Research in Science Teaching, Dallas TX, 2005 (unpublished).

2. E.L. Throndike and R. S. Woodworth, Psych. Rev. 8, 247-261 (1901).

3. C.H. Judd, Ed. Rev. 36, 28-42 (1908).

4. K. Signley, J.R. Anderson, The Transfer of Cognitive Skill (Harvard UP, Cambridge MA, 1989).

5. J.D. Bransford, D. Schwartz, Rev. of Res. Edu, 24, 61100 (1999).

6. J.G. Greeno, J.L. Moore, D.R. Smith, in Transfer on trial: Intelligence, cognition and instruction, edited by D.K. Detterman and R.J. Sternberg (Norwood, NJ, 1993) p. 99-167.

7. J.E. Lobato, Ed. Res, 32, 1, 17-20 (2003).

8. D. Schwartz, J.D. Bransford, D. Sears in Transfer of Learning form a Modern Multidisciplinary Perspective editied by J.P. Mestre (Information Age, Greenwich CT, 2005).

9. Csíkszentmihályi, Finding Flow, 1987 